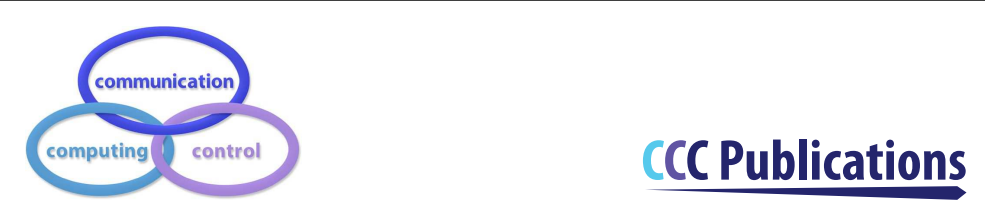

\title{
Modelling and Analysis of IoT Technology Using Neural Networks In Agriculture Environment
}

\author{
Emre Özbilge, Yönal Kırsal, Ersin Çağlar
}

\author{
Emre Özbilge* \\ Department of Computer Engineering \\ European University of Lefke, North Cyprus \\ TR-10 Mersin, Turkey \\ *Corresponding author: eozbilge@eul.edu.tr
}

\section{Yönal Kırsal}

Department of Electronics and Communication European University of Lefke, North Cyprus TR-10 Mersin, Turkey ykirsal@eul.edu.tr

\section{Ersin Çă̆lar}

Department of Management Information Systems

European University of Lefke, North Cyprus

TR-10 Mersin, Turkey

ecaglar@eul.edu.tr

\begin{abstract}
The rapid development of internet, cloud computing and sensor networks lead to develop and deploy the Internet of Things (IoT) which is a hot topic for the researchers. It has started to be used in various areas. Thus, agriculture is one of the most popular IoT research area. In agriculture environment, farming platform area is being a huge open structure and farmers must protect the crops from extreme weather conditions namely; wind speed/direction, precipitation, air temperature, solar radiations, and relative humidity etc. These extreme weather conditions effect crops and farms very significantly. But with the benefits of Internet of Things technologies, an agriculture business become more easy and efficient despite extreme weather conditions. This paper provides a model of smart agriculture environment using neural networks that helps the farmers to make more accurate predictions for the future according to weather conditions. This paper proposed a time-delay radial basis function (TDRBF) network approach to model temporal and sequential relationship between the various weather condition sensor readings from the agricultural environment. The performance of the acquired network model was analysed statistically and presented in this paper. As a result, the results of the neural network model show that it could be used to predict the desired weather condition sensor readings beforehand in order to increase the productivity in agricultural environment and also it is possible that by using such an intelligent learning system could provide a life-long learning for the changing weather conditions in the farming area over the years.
\end{abstract}

Keywords: neural networks, internet of things, agriculture, machine learning, intelligent systems. 


\section{Introduction}

Due to the improvement of the internet technology, many technologies came to our daily life such as Cloud Computing, Green Computing and Internet of Things (IoT) etc. IoT is the most popular technology due to its wide range of applications. The terminology of IoT was proposed in 1999 by Kevin Ashton as an interconnected devices [1]. As a definition, IoT is the network of physical devices that enables these devices to collect and exchange data. IoT has two important parts as a hardware and software. Hardware can be any object such as vehicles, furniture, building, home appliance with an embedded sensors. Software is the framework that creates connection with devices to identify and communicate each other [21]. With this unlimited connectivity as well as capacity, IoT has a lot of application areas. Thus, agriculture is one of the application areas too. Agriculture is the backbone of business sector for every country. In addition, agriculture is the basis for the human being as it is the main source of food and it plays important role in the growth of countries economy. The changing of natural factors such as climate, temperature, rain etc. affect all aspects of agriculture and creating many new problems all around the world [15]. The usage of IoT technology can be improved by the efficiency of overall agriculture business. Hence, this helps farmers to control unpredictable environmental factors to minimize the effects on the production cycle [27]. In order to maximize the production efficiency and the quality of the services (QoS) for agriculture environment, the environmental variables need to be constantly checked. Hence, there should be methods that able to change and/or adapt them according to the changing variables. The process of IoT applications can be realised with the combination of sensors, localization technology, processing platforms, wireless connectivity and low power devices integrated in small packages, which can be used to measure the environment variables to create an IoT agriculture system [24]. According to Gartner, approximately 25 billion identifiable devices are expected to be a part of this IoT technology by year 2020. Thus, agriculture can be a vast area to IoT with distributed autonomous sensors to monitor environmental condition of grain stores and to analyse data and exchange the information [28]. In agriculture, it is important to know the behaviour of the weather in advance, therefore, farmers are able to harvest the crops before they suffer from the bad weather conditions. In order to estimate these conditions, artificial neural network is such a powerful tool to model the non-linear weather data. In this paper, a Time-Delay Radial Basis Function (TDRBF) network [5] is proposed for predicting the future expected weather sensor readings. This network uses time-delay inputs, so that, it is capable of learning the relationship between the past and future weather sensory readings. The aim of this paper is to implement IoT technology in agriculture to analyse the unpredictable environment factors and help farmers to increase productivity. The structure of the paper is as follows: Section 2 explains the researches done in the past, Section 3.1 describes the experimental setup of the agricultural environment, Section 3 presents the overview of the proposed system for agriculture. In Section 4, a neural network approach and its learning algorithm used in this study is demonstrated and afterwards the experimental results of the proposed system using real world data is given in the Section 5. Finally, Section 6 points out some conclusion and suggests related future work.

\section{Literature review}

Agriculture is an important sector in any country and it plays a crucial role for economic growth [2]. Hence, because of this reason, agriculture environments should be supported with latest technology to get much more benefit. In literature, different technologies have been used by the researchers depending on these benefits. The authors in [25] proposed a polyhouse monitoring system with wireless sensor network (WSN). The proposed system measures the temperature, humidity, carbon dioxide level and detection light level of the polyhouse. On the other hand, the authors designed small greenhouse model in lab environment and used smart system in this greenhouse model as a smart solution to help farmers in [16]. The research study implemented in [22] used IoT and Cloud computing to monitor the crop production based on temperature and rainfall. Another research model proposed for real time monitoring the soil depends on temperature, moisture, $\mathrm{pH}$. and implemented decision support system for crop disease identification by using an image analysis based on SMS alert 
[20]. In another study, authors presented an empirical model with the combination of IoT and Cloud computing [9]. This approach promotes the fast improvement of agriculture and helps to realise smart solution for agriculture. With these improvement and solutions, researchers try to solve problems efficiently related to farmers [26]. On the other hand, the authors designed and implemented IoT based monitoring system with graphical user interface visualisation software for crop environment in [18]. The aim was to improve the agricultural forecast. Another management system was proposed in [23] as a smart cloud-controlled irrigation management system. The authors used sensors and actuators for monitoring water of the plants and cloud-based framework hosts a whether forecast [3]. In another research study, authors used IoT technology and image processing together to determine the environmental factors. This will help farms to protect farm field and get a better production [14]. The authors in [17] proposed a SmartFarm platform. The platform contains many technologies such as weather prediction, satellite imagery, soil and water monitoring. The main aim of this platform was to collect data effectively and efficiently. However, none of the models above have not considered neural network models to predict desired output considering the different weather conditions. Hence, in this paper, such an intelligent system is proposed and presented. The proposed model results are promising and explained in the following sections.

\section{The proposed model and methodology}

In this section, the proposed model and methodology are presented using neural networks in agricultural environment. A TDRBF network approach is used to model temporal and sequential relationship between the various weather condition sensors readings from the agricultural environment.

\subsection{Experimental setup}

The data used in this paper are taken from the North Wyke Farm data portal where they are made publicly available $[8,10,11]$. The Farm Platform provides three farming systems in farmlets and each consisting of five component catchments. Each farmlet is managed using alternative approaches to livestock production from grassland and measurements on water, air and soil are also recorded. Most of the data obtained have been received in every 15 minute in this platform. Please note that we have used only MET Station data for whole of Farm Platform for 2017 and 2018. The Farm Platform provides meteorological stations for whole system, soil and precipitation for each catchment as well as water flow and properties for each catchment. In other words, many parameters (data) are collected for usage. However, precipitation $p(t)$, air temperature at $(t)$, relative humidity $r h(t)$, wind speed $w s(t)$, wind direction $w d(t)$ and solar radiation $s r(t)$ have been used in this paper for analysis. Hence, the sample data used for analysis in this paper is given in Figure 1. Various sensors were allocated at the farming area in order to obtain data. However, please note that for the meteorology station data, it is not necessary to select a catchment from the map since the collected data is for global evaluation. The detailed information on the Farm Platform data collections and guides to their use can be found in $[8,10,11]$.

\subsection{The proposed model}

In this section, the proposed model and methodology used are explained using neural networks. Figure 2 shows an overview of the proposed system for the farm model.

Before the data was presented to the acquired model of farm, each sensory data was pre-analysed individually and the result showed that the sampling rate from the sensors was found very high. This is very important because modelling the farm parameters requires to use of relevant information from the data streaming in each time, otherwise, it is possible that the model receives same information in each consecutive time as a result of the model might fail to predict expected future values of the required farm parameters. To analyse sensory data, autocorrelation test [13] was applied separately for each sensor. Briefly, the sampling rate could be selected when autocorrelation between $x(t)$ and $x(t+\tau)$ has fallen below $e^{-1}$. 


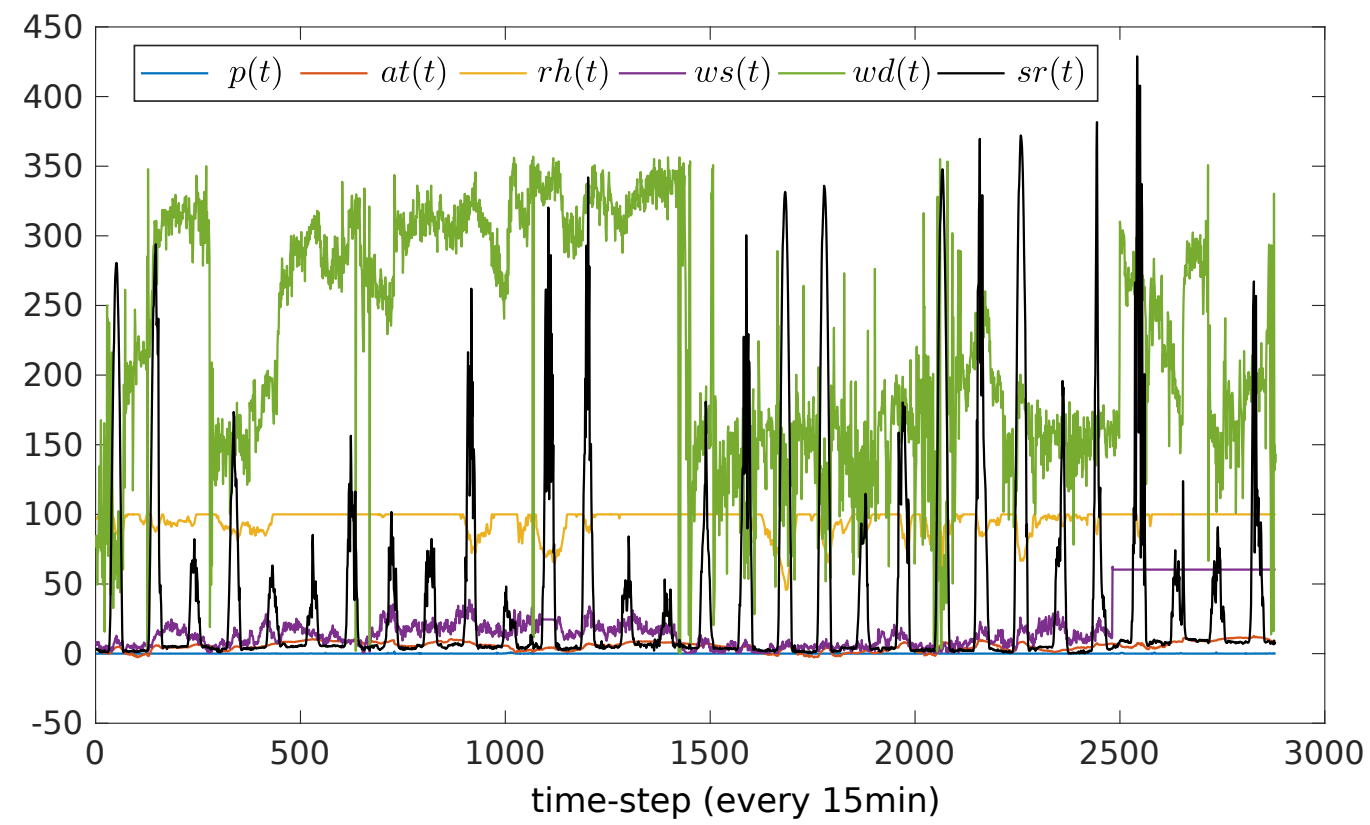

Figure 1: Sample data from real-time streaming at the North Wyke Farm platform [11]

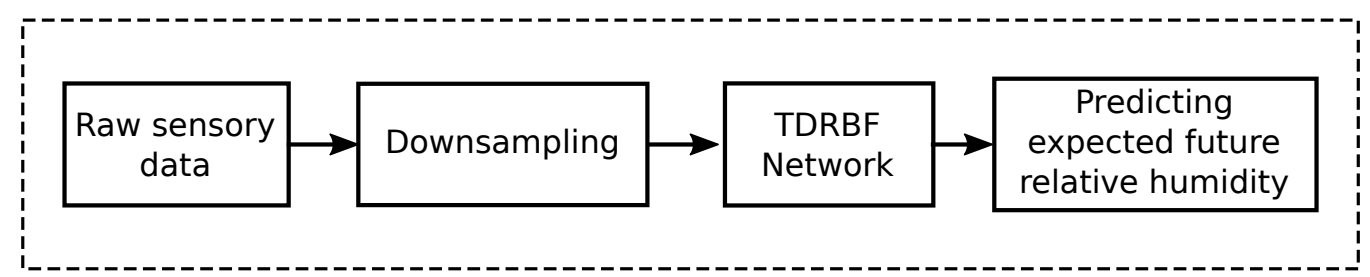

Figure 2: The proposed framework for the model of farm.

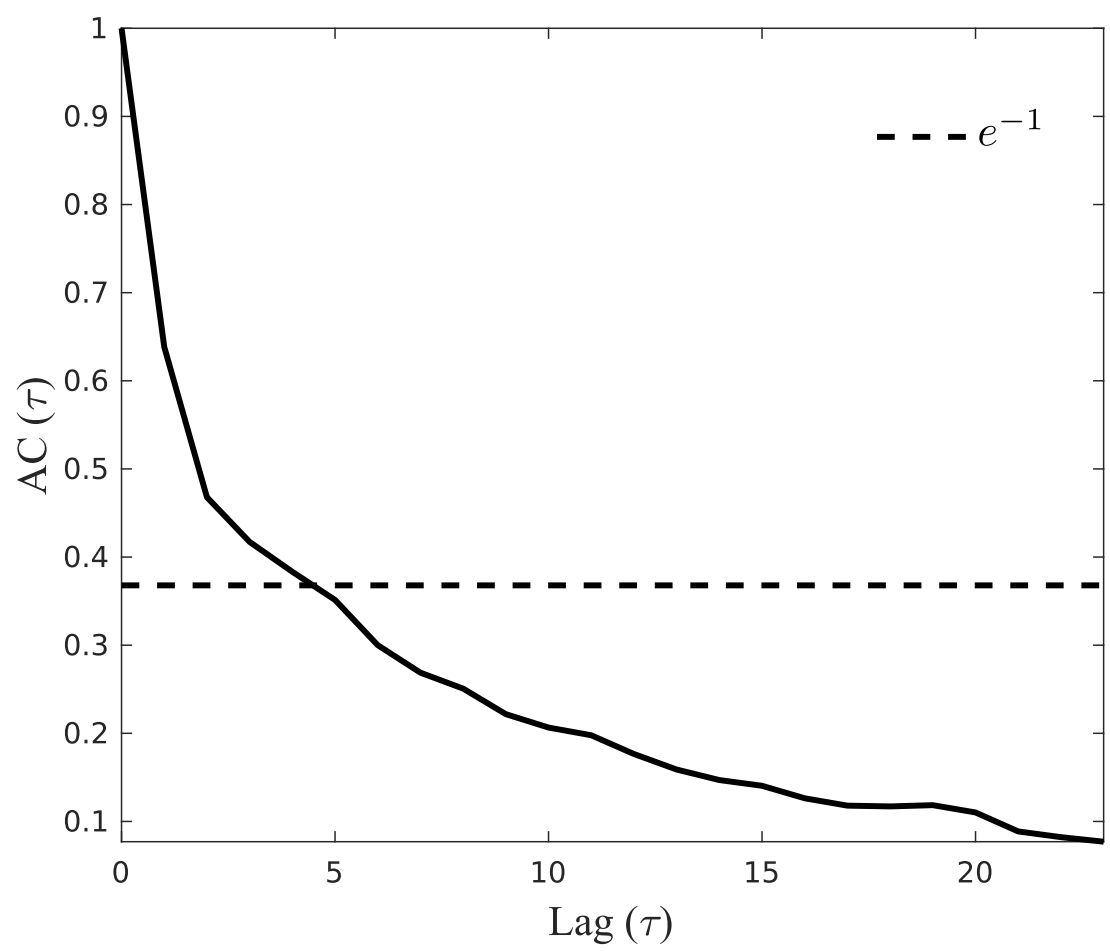

Figure 3: Autocorrelation results for the precipitation sensor where the lag equal to 5 at which has fallen below $e^{-1}$. 
Due to use of all sensors data at the same time as each an input to the farm model, the minimum lagged sensor data was selected as the down-sampling rate of all the sensors. The result of this analysis was given in Figure 3 where the precipitation data was found that the relevant data was received in every five time-steps. Consequently, the sensors data were down-sampled by taking the average of the every five consecutive streamed data so that the sampling rate became 75 minute after down-sampling process.

Afterwards, the down-sampled data was presented to a TDRBF network. Figure 4 shows the inputs and output configuration of the TDRBF network for this experiment. Various sensors were allocated at the farming area, these are precipitation $p(t)$, air temperature at $(t)$, relative humidity $r h(t)$, wind speed $w s(t)$, wind direction $w d(t)$ and solar radiation $s r(t)$ sensors as clearly seen in the Figure 4 . The data from these sensors were sampled every 15 minute. The network receives six inputs which are the data from six sensors and then predicts the expected future value of the relative humidity. Here, it is important to note that the proposed farm model network predicts 1-week ahead desired relative humidity sensor values.

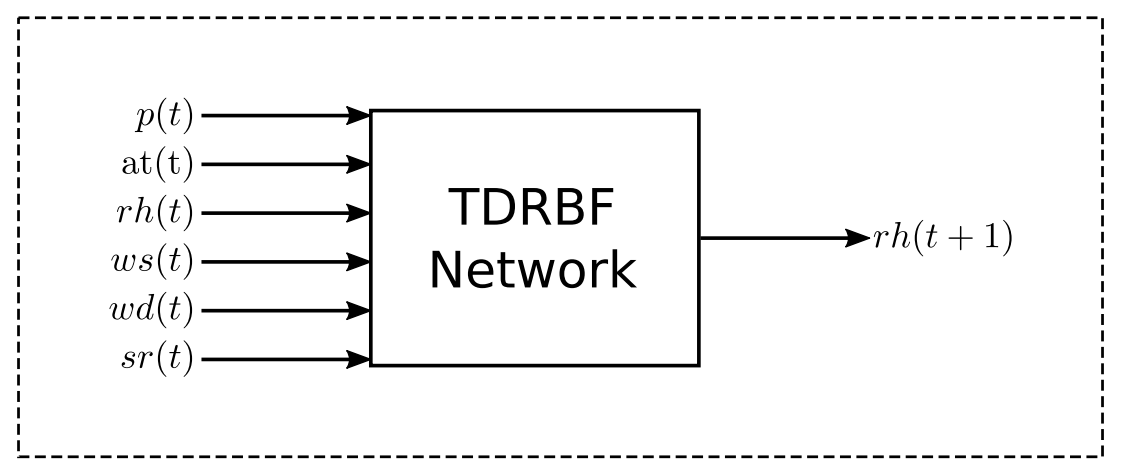

Figure 4: Inputs and outputs of the TDRBF network model for predicting the expected future value of the desired sensor.

\section{TDRBF network model}

The radial basis function (RBF) network has been commonly used for function approximation and classification problems [6, 12]. The RBF network consists of two layers; hidden and output layers. A hidden layer represents a number of nodes often each node is called a kernel which perform a nonlinear transformation. The kernel is usually a Gaussian function which is defined with two specific parameters; center vector and a width of the function. The inputs of the network are first presented to the hidden layer, then the output of the network is calculated by simply integrating the weighted outputs of the normalised hidden nodes linearly. One limitation of using standard RBF network in the farm experiment is that the network cannot learn the temporal or sequential dependencies in order to predict the future output of the desired sensor. To overcome this limitation, time delayed inputs are also added to the inputs of the network so that the network is capable of learn the relationship between the past sensory data and the future desired sensor. An overview of the TDRBF network is shown in Figure 5.

The network receives the normalised sensory inputs $\mathbf{x}(t):[p(t), a t(t), r h(t), w s(t), w d(t), s r(t)]$ in the range of $[0,1]$ at time $t$. Then, time delayed input vector is constituted by storing the actual input vector $\mathbf{x}(t)$ with $n_{x}$ time-delays:

$$
\mathbf{z}(t)=\left[\mathbf{x}(t), \mathbf{x}(t-1), \ldots, \mathbf{x}\left(t-n_{x}\right)\right] .
$$

To compute the output of each hidden node, the Euclidean distance $\mathbf{d}(t)$ between the time delayed input vector and the center vector $\mathbf{c}_{i}$ of each hidden unit are computed as:

$$
d_{i}(t)=\left\|\mathbf{z}(t)-\mathbf{c}_{i}\right\|=\sqrt{\sum_{j=1}^{k}\left(z_{j}(t)-c_{i, j}\right)^{2}},
$$




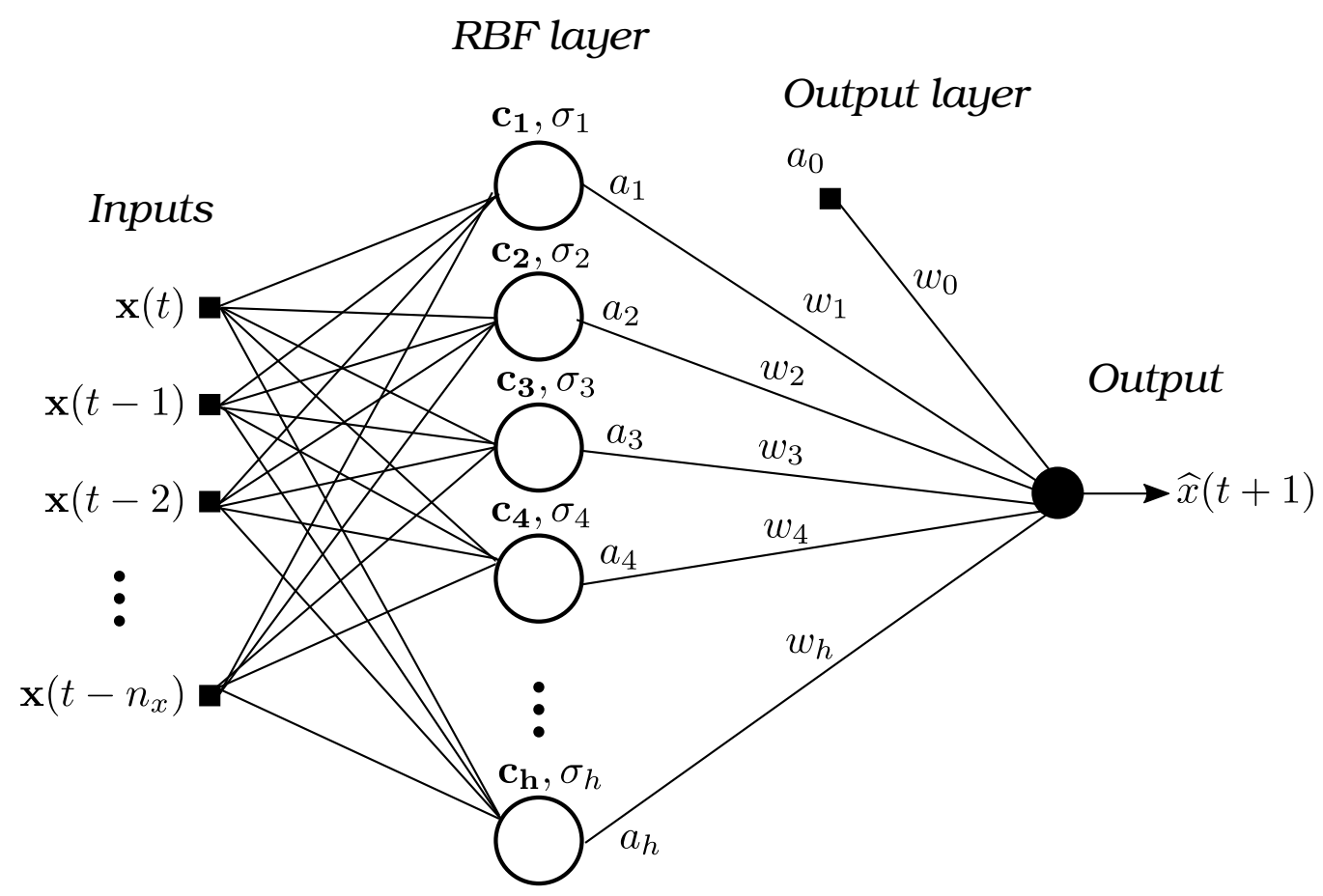

Figure 5: The structure of TDRBF network.

where $i$ is an index of the current hidden node $(i=1, \ldots, h, h$ is the number of hidden nodes), $k=n \cdot\left(n_{x}+1\right)$ represents the dimensionality of the center vector, here $n=6$ indicates the number of sensory inputs and $c_{i, j}$ is the center from the time delayed input $j$ to the hidden node $i$.

The output of the hidden nodes $\mathbf{a}(t)$ is then calculated using a normalised Gaussian function.

$$
a_{i}\left(d_{i}(t), s_{i}\right)=\frac{\exp \left(-\frac{d_{i}^{2}(t)}{2 \cdot \sigma_{i}^{2}}\right)}{\sum_{j=1}^{h} \exp \left(-\frac{d_{j}^{2}(t)}{2 \cdot \sigma_{j}^{2}}\right)},
$$

where $\sigma_{i}$ is the width of hidden node $i$.

Finally, the network-predicted outputs $\widehat{\mathbf{x}}(t+1)$ for the expected future sensory input value is calculated by linearly integrating the output of the hidden nodes:

$$
\widehat{x}(t+1)=\sum_{i=0}^{h} w_{i} \cdot a_{i},
$$

where $w_{i}$ is the connection weight from hidden node $i$ to the output, $w_{0}$ indicates the bias term and $a_{0}=1$.

\subsection{Training TDRBF network}

Training of the TDRBF network requires two stages of learning. Firstly, center vectors $\mathbf{c}_{i}(i=$ $1,2, \ldots, h)$ of the hidden nodes are trained by using $k$-means algorithm. After having obtained center vectors, the width of each hidden node $\sigma_{i}$ is computed using the $p$-nearest neighbours heuristic approach [4]:

$$
\sigma_{i}=\frac{1}{p} \cdot \sqrt{\left(\sum_{j=1}^{p}\left\|\mathbf{c}_{i}-\mathbf{c}_{j}\right\|^{2}\right)}, \forall i \neq j,
$$

where $\mathbf{c}_{j}$ represents the $p$-nearest neighbours center vector to the current center vector $\mathbf{c}_{i}$ and the parameter $p$ is set to be 2 . 
Afterwards in the second learning stage, the normal equation solution with regularisation is applied to obtain the weights of the output layer w. Summary of the training algorithm of the TDRBF network is given in Algorithm 1.

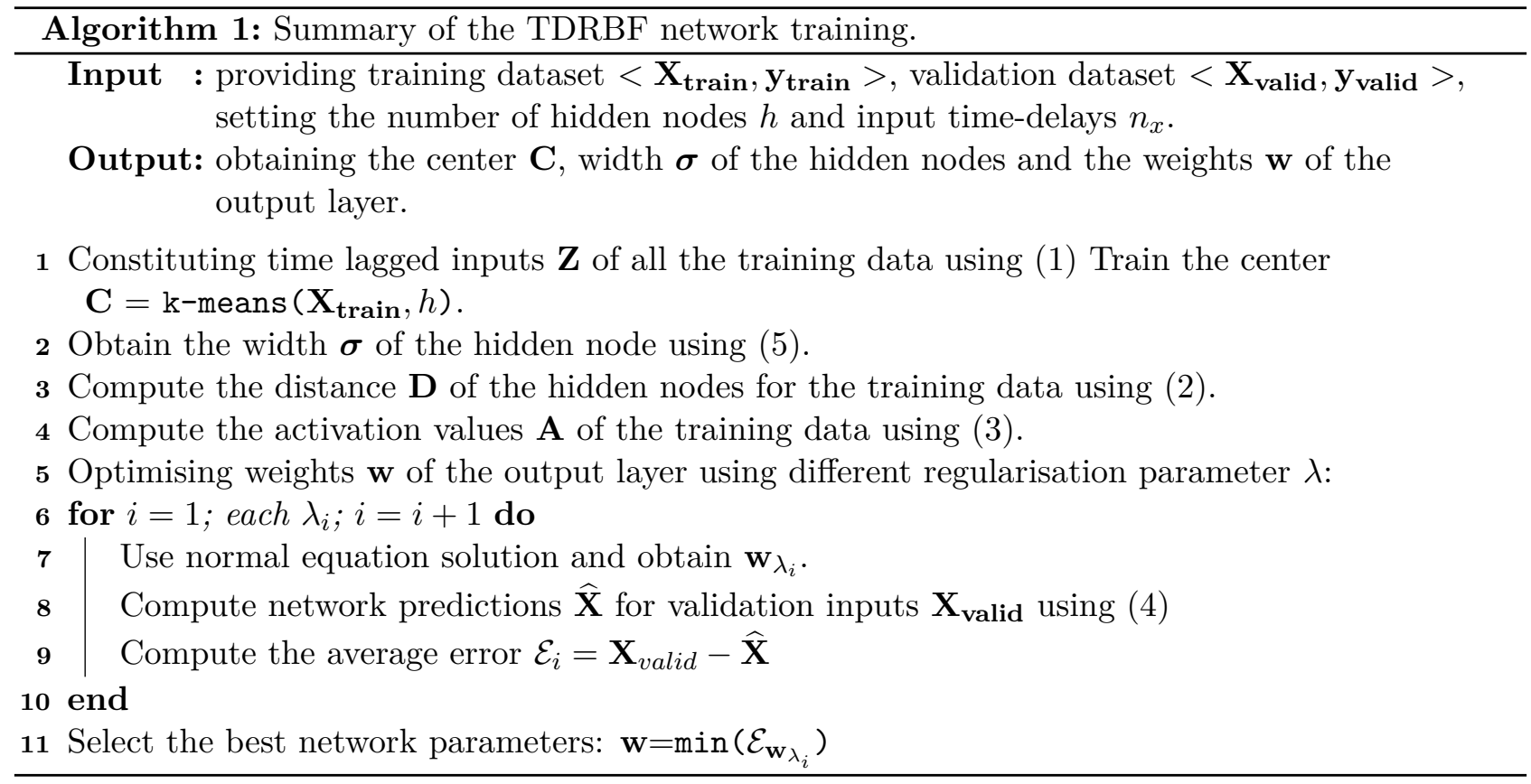

\subsection{Parameter selection}

There are two important parameters which have to be defined, these are time delay parameter $n_{x}$ and the number of the hidden nodes $h$. To predict 1 -week ahead value of the relative humidity sensor, the network requires sufficient number of past input sensory data according to model the future value of the desired sensor. Therefore, the training parameter $n_{x}$ was set to be 2 -weeks of data points receiving from the sensors in the farming area. To create 2-weeks memory for TDRBF network, simply $n_{x}=19 \times 14=266$ past data points were used where 1-day data information consists of 19 data points and 14 represents the number of the day here. More time delayed input data points could be added to the network but it could cause the network unable to generalise unseen sensory data and also less time delayed input data outcomes unable to model the temporal or sequential sensory informations [7]. In this case, the network predicts $19 \times 7=133$ data points ahead value of the desired sensor which implies 1-week ahead network-prediction. It is also essential to choose the right number of hidden node for the neural network training. Because selecting a small or a large number of hidden node can lead underfitting or overfitting problem respectively. Hence to define the parameter $h$, different number of hidden node were tested for the network eventually 1500 hidden nodes yielded the best performance in this case. In the neural network literature, to find the optimal number of hidden nodes, there are various techniques, however, this is not the purpose of this paper.

\section{$5 \quad$ Experimental results}

After the network was trained using 1-year of data from the farming environment, the obtained farm model was tested using unseen data which were logged from the other year.

The result of the network predictions is shown in Figure 6. It is clearly seen that there is some small fluctuating between the actual and predicted relative humidity values. This is expected result because the network predicts future values of the desired sensor instead of nearer expected values. To assess the quality prediction performance of the network, Pearson's correlation coefficient was computed and the correlation coefficient between the actual and predicted values was found to be 0.8 (note that coefficient value 1 indicates perfect matching). 


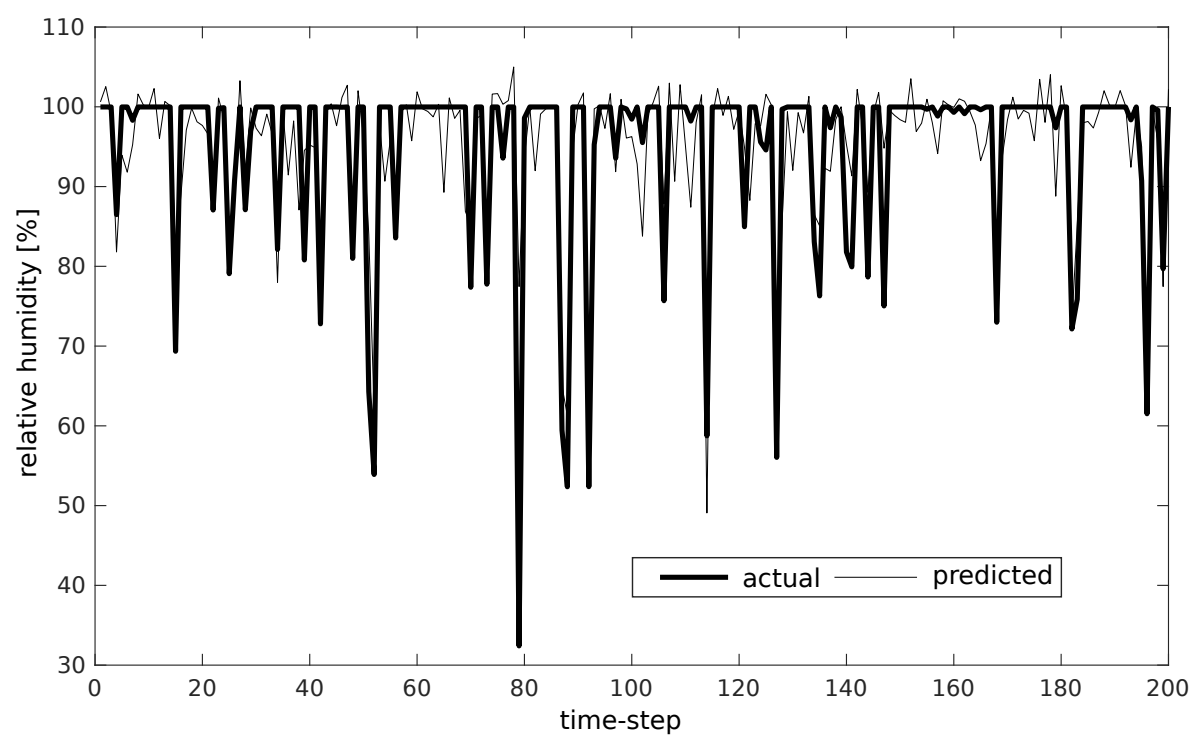

(a)

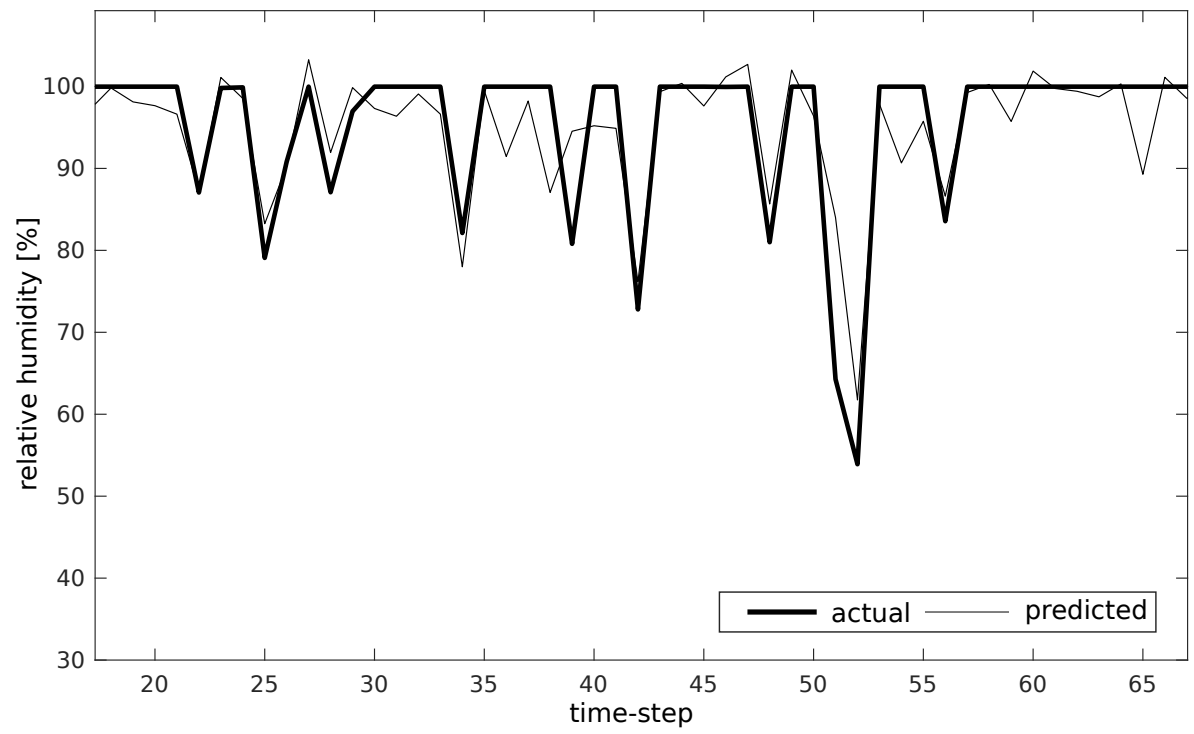

(b)

Figure 6: (a) Actual relative humidity sensor data and the network-predicted values of the sensor. Total of 5455 data points were tested. For the clarity, only the first 200 test data points are displayed on the graph. (b) Graph is zoomed to display more detail predicted versus actual sensory readings about time-steps between 20 and 65 . 


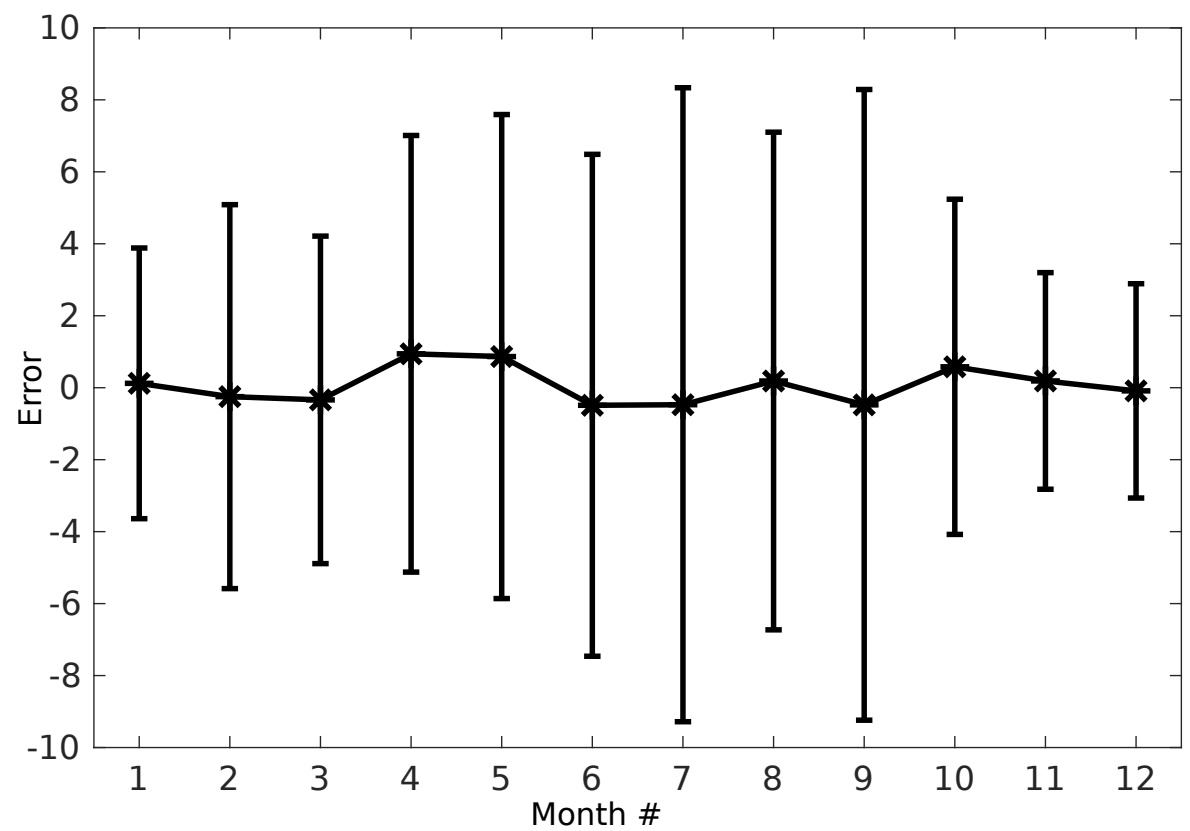

(a)

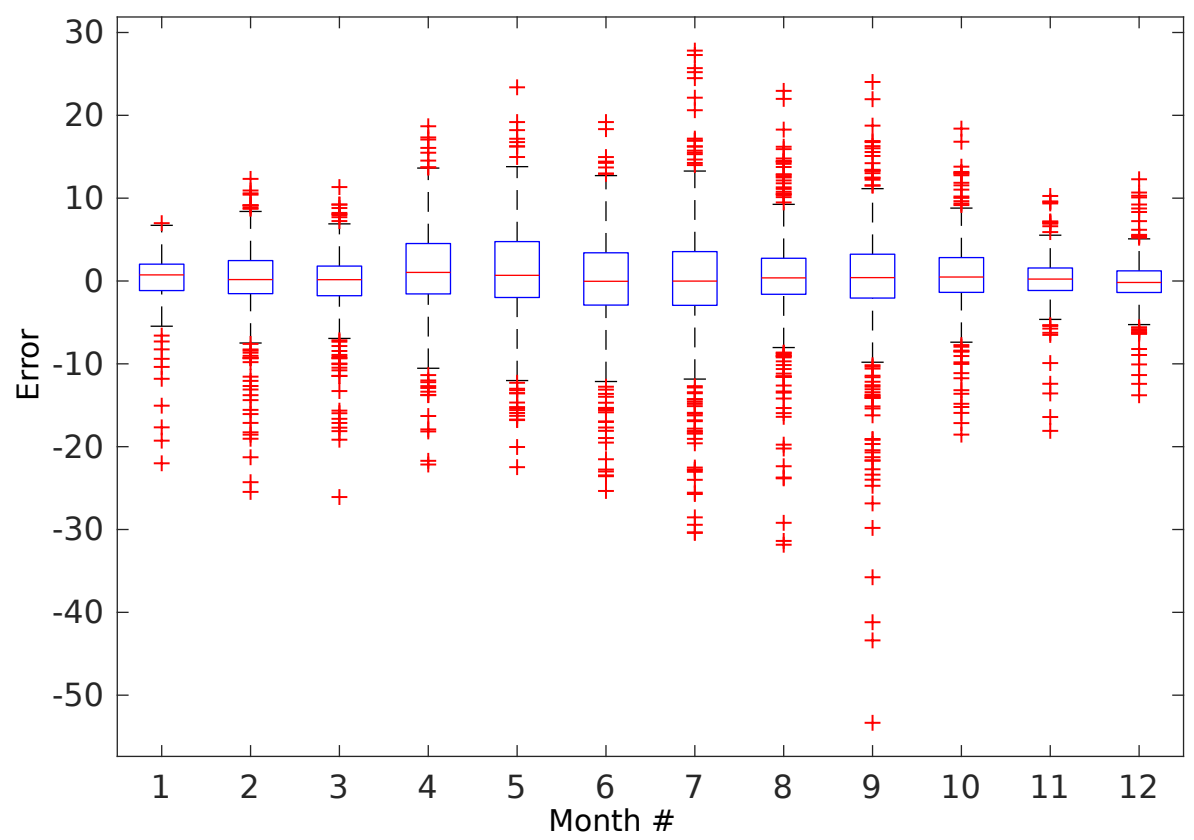

(b)

Figure 7: (a) Error bar, and (b) box plot of the prediction error. 
Furthermore, t-test was also applied to the network residual error and it was found that the mean value of the residual error is statistically similar to zero at the $1 \%$ significance level. In addition, to analyse how much deviation between the model-predicted outputs and the actual relative humidity sensor readings occurred, confidence interval of the mean of the residual error is also computed at the same significance level and was between $[-0.24,0.17]$. Further analyses were also carried out in order to understand deeply the behaviour of the neural network model on the farm data in the course of the predicted year. Figure 7 shows the error bar and box plot of the prediction errors associated with each month of the predicted year. It is clearly seen that the acquired network model showed varied performances in comparison between the months of the corresponding year. These uncertainties were shown with the standard deviations of the prediction errors of each month as given in Figure 7a. Especially, the month 7 and 9 (i.e. July and September) are the highest deviation occurred from their corresponding mean error with approximately 8.8 standard deviation. Similar results can be also seen in the Figure $7 \mathrm{~b}$ where the end of the whiskers, in the case of July and September, indicate the maximum and minimum distances to the median error (median error is the red line in the box) and these values are the largest in comparison with other months. Surely in month 4, 5 and 6 (April, May and June) in the Figure $7 \mathrm{~b}$ have similar maximum and minimum distances to the their corresponding median error but the number of the outliers that are indicated with plus symbols, are lesser than the month 7 and 9 . As a result, the volume of the outliers increased the standard deviation of the month 7 and 9. In addition, although the month 8 (August) has lower minimum and maximum distances to its median error, the volume of the outliers are high, so that it has similar standard deviation with month 4,5 and 6 .

The input data of July and September from the training and testing dataset were examined statistically whether or not both datasets are from same distribution. Thus, Kolmogorov-Smirnov (KS) test was applied to compare the training and testing data of each sensor on the network. The results showed that; the null hypothesis was rejected for all the sensory inputs data between the training and testing sets at the 5\% significance level in July. Similarly for the September, only the wind speed $w s$ sensor data from the training and testing sets come from same distribution and the null hypothesis was rejected for the other sensory data. This was expected results due to the high standard deviations for the both months. However, we believe that uncertain sensory data is normal between years due to the fact that global warming. Nevertheless, the acquired TDRBF network generalised well on the noisy sensory data from the farming area.

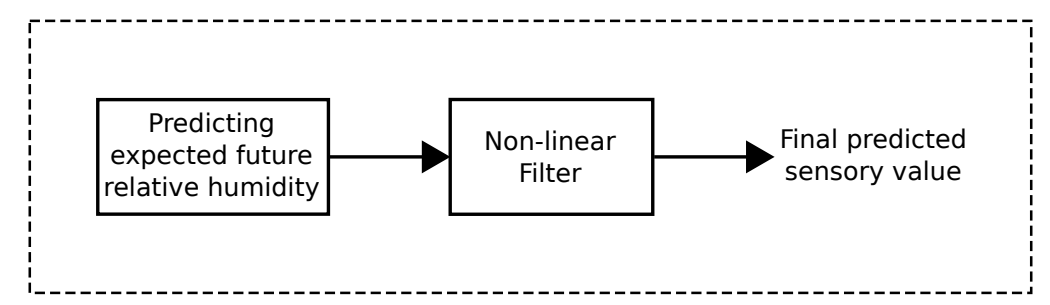

Figure 8: Possible extension for the proposed framework of the model of farm.

One way to overcome the problem of the noisy data is that to extend the proposed framework given in Figure 2 with a non-linear filter [19]. Therefore, after the network predicts the future value of the corresponding sensors, the raw predicted values pass through the some smoothing processes by using previously predicted values before the final decision on the predicted sensory values. This extension is illustrated in Figure 8.

\section{Conclusion and future work}

In this paper, a TDRBF network-based agricultural weather data forecasting model was demonstrated. The steps of the proposed agricultural TDRBF model-based framework was described in section 3. Due to the high sampling rate from the sensors in the agricultural area, down-sampling process was suggested which was found out in autocorrelation analysis given in Figure 3. Afterwards, the structure of TDRBF network, training algorithm and the parameter selection approach for the agri- 
cultural problem were described in section 4 . The TDRBF network performed very promising results for the predictions of the expected future weather sensor values. To verify the network performance, statistical analyses were also carried out in section 5 and the prediction errors between the obtained network model and the actual sensor readings were found very low at the $1 \%$ significance level. In addition, Figure 6 proved that how robust the network predictions on the unseen noisy weather sensor data. Consequently, the generalisation capability of the network was the desired outcomes for the globally changing weather data over the years in the world. For the future work, the study can be further explored in many direction as follows;

- Comparison of the different type of neural networks for the same scenario;

- Developing a new region-based agricultural weather forecasting framework, so that, for the different regions, a specific sensors network model will be selected for generating more reliable future predictions. This is because in the large agricultural environment, it is possible that weather sensors readings could be different from each other which are located at the different regions in the same environment.

\section{Acknowledgement}

The authors are deeply grateful to North Wyke Farm Platform and Rothamsted Research in providing of the data publicly available.

\section{Author contributions}

The authors contributed equally to this work.

\section{Conflict of interest}

The authors declare no conflict of interest.

\section{References}

[1] Ashton, K. (2009). That 'internet of things' thing, RFID journal, 22(7), 97-114, 2009.

[2] Awuor, F.; Kimeli, K.; Rabah, K.; Rambim, D. (2013). ICT solution architecture for agriculture, In 2013 IST-Africa Conference \& Exhibition, IEEE, 1-7, 2013.

[3] Ayaz, M.; Ammad-Uddin, M.; Sharif, Z.; Mansour, A.; Aggoune, E.H.M. (2019). Internet-ofThings (IoT)-Based Smart Agriculture: Toward Making the Fields Talk, IEEE Access, 7, 129551129583, 2019.

[4] Benoudjit, N.; Verleysen, M. (2003). On the kernel widths in radial-basis function networks, Neural Processing Letters, 18(2), 139-154, 2003.

[5] Berthold, M.R. (1994). A time delay radial basis function network for phoneme recognition, In Proceedings of 1994 IEEE International Conference on Neural Networks (ICNN'94), IEEE, 7, 4470-4472, 1994.

[6] Bishop, C.M. (1995). Neural networks for pattern recognition, Oxford University Press, 1995.

[7] Boden, M. (2002). A guide to recurrent neural networks and backpropagation, The Dallas Project, 2002.

[8] Griffith, B.A.; Hawkins, J.M.B.; Orr, R.J.; Blackwell, M.S.A.; Murray, P.J. (2013). The North Wyke Farm Platform: methodologies used in the remote sensing of the quantity and quality of drainage water, In Revitalising Grasslands to Sustain our Communities: Proceedings, 22nd International Grassland Congress, 15-19 September, 2013, New South Wales Department of Primary Industry, Sydney, Australia, 1453-1455, 2013. 
[9] Haque, M.S.T.; Rouf, K.A.; Khan, Z.A.; Emran, A.; Zishan, M.S.R. (2019). Design and Implementation of an IoT based Automated Agricultural Monitoring and Control System, In 2019 International Conference on Robotics, Electrical and Signal Processing Techniques (ICREST), IEEE, 13-16, 2019.

[10] Harris, P.; Sint, H.M.; Griffith, B.A.; Hawkins, J.M.B.; Evans, J.; Orr, R.J.; Lee, M.R.F. (2016). The North Wyke Farm Platform: data portal, in The multiple roles of grassland in the European bioeconomy, Proceedings of the 26th General Meeting of the European Grassland Federation, Trondheim, Norway, NIBIO, 618-620, 2016.

[11] Harrod, T.R.; Hogan, D.V. (2008). The soils of North Wyke and rowden. Soil survey of England and Wales, Rothamsted Research, Okehampton, 2008.

[12] Haykin, S. (1994). Neural networks: a comprehensive foundation, Prentice Hall PTR, 1994.

[13] Kaplan, D.; Glass, L. (2012). Understanding nonlinear dynamics, Springer Science \& Business Media, 2012.

[14] Kapoor, A.; Bhat, S.I.; Shidnal, S.; Mehra, A. (2016). Implementation of IoT (Internet of Things) and Image processing in smart agriculture, In 2016 International Conference on Computation System and Information Technology for Sustainable Solutions (CSITSS), IEEE, 21-26, 2016.

[15] Khattab, A.; Abdelgawad, A.; Yelmarthi, K. (2016). Design and implementation of a cloud-based IoT scheme for precision agriculture, In 2016 28th International Conference on Microelectronics (ICM), IEEE, 201-204, 2016.

[16] Kodali, R.K.; Jain, V.; Karagwal, S. (2016). IoT based smart greenhouse, In 2016 IEEE Region 10 Humanitarian Technology Conference (R10-HTC), IEEE, 1-6, 2016.

[17] Krintz, C.; Wolski, R.; Golubovic, N.; Lampel, B. et. all (2016). SmartFarm: Improving agriculture sustainability using modern information technology, In KDD Workshop on Data Science for Food, Energy, and Water, 2016.

[18] Lee, M.; Hwang, J; Yoe, H. (2013). Agricultural production system based on IoT, In 2013 IEEE 16Th international conference on computational science and engineering, IEEE, 833-837, 2013.

[19] Mallows, C.L. (1980). Some theory of nonlinear smoothers, The Annals of statistics, 8(4), 695-715, 1980.

[20] Patil, K.A.; Kale, N.R. (2016). A model for smart agriculture using IoT, In 2016 International Conference on Global Trends in Signal Processing, Information Computing and Communication (ICGTSPICC), IEEE, 543-545, 2016.

[21] Patil, V.C.; Al-Gaadi, K.A.; Biradar, D.P.; Rangaswamy, M. (2012). Internet of things (Iot) and cloud computing for agriculture: An overview, Proceedings of agro-informatics and precision agriculture (AIPA 2012), India, 292-296, 2012..

[22] Rajesh, D. (2011). Application of spatial data mining for agriculture, International Journal of Computer Applications, 15(2), 7-9.

[23] Sales, N.; Remédios, O.; Arsenio, A. (2015). Wireless sensor and actuator system for smart irrigation on the cloud, In 2015 IEEE 2nd World Forum on Internet of Things (WF-IoT), IEEE, 693-698, 2015.

[24] Sisinni, E.; Saifullah, A.; Han, S.; Jennehag, U.; Gidlund, M. (2018). Industrial internet of things: Challenges, opportunities, and directions, IEEE Transactions on Industrial Informatics, 14(11), 4724-4734, 2018.

[25] Song, Y.; Ma, J.; Zhang, X.; Feng, Y. (2012). Design of wireless sensor network-based greenhouse environment monitoring and automatic control system, Journal of Networks, 7(5), 838, 2012. 
[26] Srinivasan, G.; Vishnu Kumar, N.; Shafeer Ahamed, Y.; Jagadeesan, S. (2017). Providing smart agricultural solution to farmers for better yielding using IoT, Int. J. Adv. Sci. Eng. Res, 2(1), 2017.

[27] Tsai, C.W.; Lai, C.F.; Chiang, M.C.; Yang, L.T. (2013). Data mining for internet of things: A survey, IEEE Communications Surveys \& Tutorials, 16(1), 77-97, 2013.

[28] [Online]. Available: https://www.gartner.com/en/newsroom/press-releases/2017-02-07-gartnersays-8-billion-connected-things-will-be-in-use-in-2017-up-31-percent-from-2016, Accesed on 22 December 2019.

\section{(c) (i) \&}

Copyright (C) 2020 by the authors. Licensee Agora University, Oradea, Romania.

This is an open access article distributed under the terms and conditions of the Creative Commons Attribution-NonCommercial 4.0 International License.

Journal's webpage: http://univagora.ro/jour/index.php/ijccc/

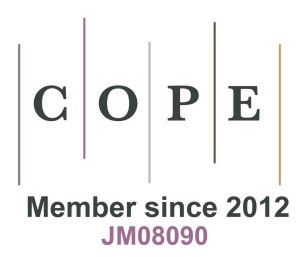

This journal is a member of, and subscribes to the principles of, the Committee on Publication Ethics (COPE).

https://publicationethics.org/members/international-journal-computers-communications-and-control

Cite this paper as:

Özbilge, E.; Kırsal, Y.; Çağlar, E. (2020). Modelling and Analysis of IoT Technology Using Neural Networks In Agriculture Environment, International Journal of Computers Communications \& Control, 15(3), 3885, 2020.

https://doi.org/10.15837/ijccc.2020.3.3885 